\title{
Relato de Caso em Cardiologia
}

\section{Miocardiopatia não compactada - relato de caso}

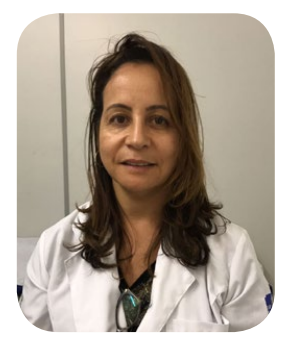

\author{
Alba Fátima Rodrigues Lima1', Thiago Carvalho Pereira1, \\ Emilly Virgínia Costa Borges²
}

\begin{abstract}
Palavras-chave: cardiomiopatias; insuficiência cardíaca; disfunção ventricular Key-words: cardiomyopathies; heart failure; ventricular dysfunction
\end{abstract}

\section{INTRODUÇÃO}

As miocardiopatias representam o segundo maior grupo de causas de morte súbita cardíaca. A miocardiopatia não compactada (MNC) tem uma prevalência de $1 / 10.000$ adultos $^{1}$, acometendo mais o sexo masculino $^{2}$. Relatada primeiramente por Chin e cols. ${ }^{3} \mathrm{em}$ 1990, trata-se de uma doença congênita, decorrente de uma anormalidade da morfogênese endomiocárdi$\mathrm{ca}^{4}$, classificada como cardiomiopatia primária geneticamente determinada pela Associação Americana de Cardiologia ${ }^{5}$ ou como miocardiopatia não classificada pela Organização Mundial da Saúde ${ }^{6}$.

Durante a fase inicial do desenvolvimento embrionário, o coração consiste em uma trama de fibras musculares, separadas por espaços, chamados intertrabeculares (ou sinusoides). Entre a $5^{\mathrm{a}}$ e $8^{\mathrm{a}}$ semana, as fibras do miocárdio se compactam e a circulação coronariana se desenvolve. $\mathrm{Na} \mathrm{MNC}$, não ocorre esta compactação das fibras, formando uma rede frouxa de fibras musculares entrelaçadas, caracterizada por uma trabeculação miocárdica excessiva ${ }^{4,7}$. A causa ainda não é totalmente compreendida, porém acredita-se que a sobrecarga de pressão ou isquemia miocárdica tenham papel fundamental na regressão dos sinusoides embrionários e consequente persistência dos espaços intertrabeculares. A MNC pode ser geneticamente esporádica ou familiar ${ }^{4}$.

As manifestações clínicas são amplas. A maioria dos pacientes apresentarão, ao longo dos anos, sintomas de falência ventricular ${ }^{4}$. Entretanto, podem ser assintomáticos ou apresentar sintomas de insuficiência cardíaca, fenômenos tromboembólicos e arritmias cardíacas, podendo ter início em qualquer idade ${ }^{4,2}$.

$\mathrm{O}$ diagnóstico da MNC é frequentemente realizado pela ecocardiografia. Os critérios mais utilizados foram propostos por Jenni et. al.8: (1) Ausência de anormali- dades cardíacas coexistentes, (2) Estrutura miocárdica de dupla camada (miocárdio não compacto e miocárdio compacto), com razão, no final da sístole, > 2:1; (3) Localização das trabéculas geralmente nas paredes apical/lateral, média/inferior do ventrículo esquerdo. (4) Fluxo entre os recessos intertrabeculares, identificados pelo doppler colorido. Os achados eletrocardiográficos não possuem alterações específicas. As anormalidades mais encontradas são: bloqueios de ramo e arritmias, como fibrilação atrial e taquicardia ventricular ${ }^{4}$.

Diferentes genes têm sido identificados. Foram descritas mutações que codificam as proteínas sarcoméricas, do citoesqueleto e da membrana nuclear, incluindo: G4.5, TAZ, PRDM16, TNNT2, LDB3, MYBPC3, MYH7, ACTC1, TPM1, MIB1 e DTNA2.

$O$ presente relato descreve quadro de paciente com MNC há 10 anos, com ênfase sobre os aspectos clínicos, terapêuticos e relação familiar.

\section{RELATO DE CASO}

Paciente do sexo feminino, 39 anos, parda, técnica em contabilidade, G1P1A0. Diagnosticada com MNC há 10 anos. Nos antecedentes: mãe falecida por morte súbita; tia falecida aos 14 anos por morte súbita; possui cinco irmãos, dos quais três possuem a doença comprovada, todas do sexo feminino.

Relato de infância e início da vida adulta assintomática, com gravidez sem intercorrências. Em 2 meses de pós-parto, evoluiu com edema em membros inferiores e ascite importante, associado à dispneia e fadiga. Feita a investigação e de acordo com critérios clínicos e identificação em ecocardiograma doppler e ressonância magnética, diagnosticou-se miocárdio não compactado. Seguiu em tratamento medicamentoso com uso de anticoagulantes e betabloqueadores. Após 1 ano, implantou o cardiodesfibrilador implantável (CDI). 
Após 6 anos, em 2015, foi submetida à troca de gerador do CDI. Neste período, sofreu acidente vascular cerebral isquêmico (AVCi), devido a trombo em artéria cerebral média. Identificado imediatamente, realizou trombólise e posterior reabilitação de sequelas com terapeuta durante 9 meses, seguindo sem sequelas.

Entretanto, após 2 anos, em 2017, os sintomas de fadiga e dispneia voltaram, associados a ascite, edema nos membros inferiores, enjoos e vômitos, levando-a a procurar o serviço de cardiologia do Hospital Santa Izabel (HSI). Exame físico de entrada: regular estado geral, consciente, orientada, ausculta pulmonar com estertores crepitantes bilateral em terço inferior, ruído cardíaco regular em 2 tempos, bulhas normofonéticas, sem sopros, edema de membros inferiores (++/4+), $P A=100 \times 70 \mathrm{mmHg}$. Eletrocardiograma: flutter atrial com condução atrioventricular 4:1 e bloqueio do ramo direito. Radiografia de tórax (Figura 1): aumento da área cardíaca, abaulamento do arco médio e parênquima pulmonar normotransparente. Ecocardiograma transtorácico (Figura 2): aumento importante de câmeras cardíacas, insuficiência tricúspide de grau importante, devido à falha de coaptação, disfunção sistólica do ventrículo esquerdo de grau importante e disfunção sistólica do ventrículo direito de grau moderado. Ultrassonografia: ascite e derrame pleural bilateral. Laboratório: elevados níveis de escórias nitrogenadas.

Encaminhado à Unidade de Terapia Intensiva Coronariana. Iniciado a terapia com: Marevan; Carvedilol; Lasix (furosemida), Aldactone (diurético poupador de potássio), Amiodarona (vasodilatador), Digoxina (digitálico) e Dobutamina. Feito cardioversão elétrica devido ao flutter atrial. Realizado troca para CDI multissítio. Evolui com melhora de função renal e das demais funções orgânicas, hemodinamicamente estável; entretanto, segue com insuficiência cardíaca em uso de Sacubitril/valsatarna em doses crescentes. Paciente sem novas complicações recebe alta em bom estado geral. Mantém acompanhamento ambulatorial.

\section{DISCUSSÃO}

No Brasil, há 300 mil casos de morte súbita decorrente de algum tipo de arritmia cardíaca por ano. A parada cardíaca súbita é uma das principais causas de morte entre os adultos com mais de 40 anos e tem sido a primeira causa de morte no mundo 9 .

As cardiomiopatias comprometem o músculo cardíaco causando disfunção mecânica e/ou elétrica. Cursa frequentemente com hipertrofia ou dilatação ventricular e falência cardíaca. A MNC possui etiologia genética. Neste caso, encontramos forte relação genética, onde foi evidenciado $83 \%$ de seus parentes do sexo feminino de primeiro grau com disfunção sugestiva ou diagnosticada por MNC. Dados estes, conflitantes com a literatura, em que relata prevalência maior do sexo masculino e recorrência familiar baixa ${ }^{2}$. Alertando que, embora relativamente rara, deve-se considerar a MNC em casos de falência ventricular, arritmia ventricular e embolizações, evitando uma triagem ineficaz e evolução da doença.

A história natural da doença não está bem estabelecida ou o porquê dos sintomas aparecem apenas aos 30 anos. Sugere-se que o período pós-parto tenha propiciado condições que evidenciassem a MNC, devido resposta imunológica a antígeno materno-fetal, em que células fetais podem escapar para a circulação materna e se alojarem no tecido cardíaco, desencadeando resposta autoimune. Condição semelhante ao observado na miocardiopatia periparto (MPP).

Geralmente, a MNC tem início insidioso, com certa hipertrofia ventricular compensatória e dilatação ventricular esquerda assintomática. Habitualmente, a intolerância aos exercícios físicos progride até insuficiência cardíaca (IC) franca, o que indica se tratar de doença progressiva. No caso, evoluiu com IC grave.

A IC é resultado de uma redução da contratilidade do miocárdio, por consequência do miocárdio não compactado. Possui características de grande volume diastólico residual, dilatação do anel valvular e insuficiência relativa das valvas mitral e tricúspide. Devido à estase de sangue nas câmeras, pode haver formação de trombos, levando a complicações sistêmicas; evidenciado, neste caso, no AVCi.

O plano terapêutico é direcionado para as manifestações mais frequentes da doença, a fim de evitar a morte súbita. Realizou-se, dessa forma, o tratamento da insuficiência cardíaca, anticoagulação profilática e avaliação do risco pró-arrítmico. A prevenção das complicações tromboembólicas possui controvérsias na literatura, sendo indicada apenas por alguns autores. Entretanto, a diretriz brasileira recomenda anticoagulação aos pacientes que apresentam função sistólica diminuída com fração de ejeção inferior a $40 \%$, história de tromboembolismo ou fibrilação atrial ${ }^{10}$, justificando o uso na paciente.

Devido à arritmia ventricular sintomática e função sistólica prejudicada, a prevenção indicada é por meio de desfibriladores cardíacos implantáveis (CDI). No caso estudado, a paciente implantou, inicialmente, o apareIho de dupla câmera. A troca para estimulação multissítio buscou melhora do sincronismo intracavitário.

Este caso ilustra a relação da MNC com a genética e reforça a necessidade de, na presença das caracte- 
rísticas destacadas, não descartar a possibilidade da doença.

\section{FIGURAS}

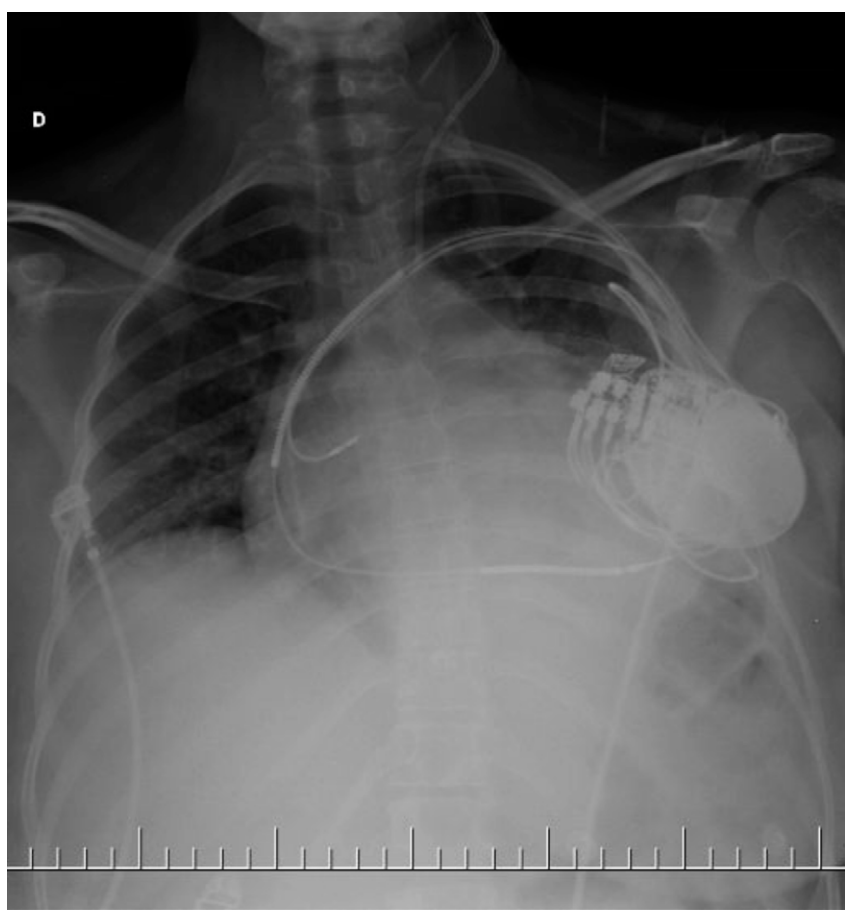

Figura 1. Radiografia de tórax em incidência posteroanterior, mostrando aumento da área cardíaca. Notar presença do CDI.

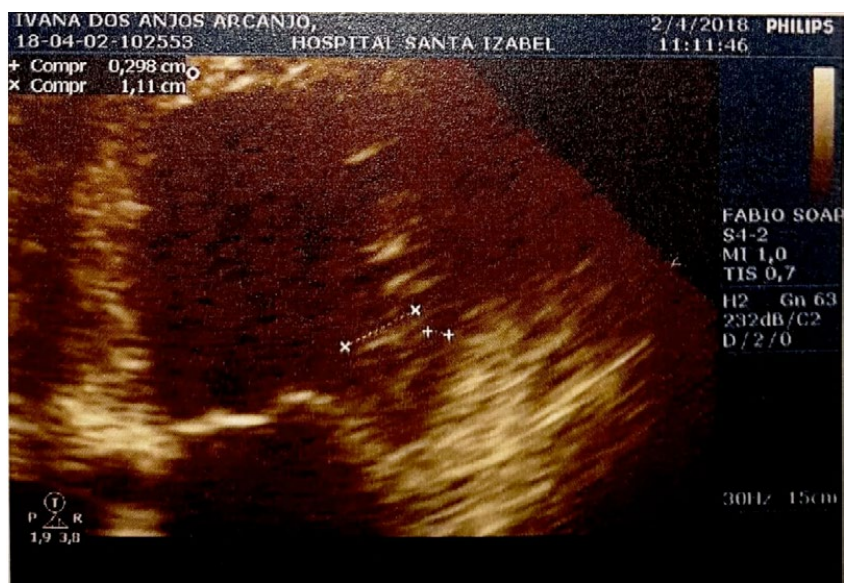

Figura 2. Ecocardiograma transtorácico. Razão miocárdio não compactado/ compactado $=3,7$ (critérios de Jenni e cols.).

\section{REFERÊNCIAS}

1. Papadakis M, Sharma S.Suddencardiac death. Medicine, 2010;38:502-506.

2. Meneguz-Moreno, $R$ et. al.. Isolated left ventricular noncompaction causing refractory heart failure. Rev Port Cardiol. 2016;35(3):185-185

3. Chin TK, Perloff JK, Williams RG, Jue J, Mohrmann R. Isolated noncompaction of left ventricular myocardium: a study of eight cases. Circulation. 1990,82(2):507-13.

4. Rosa Leonardo Vieira da, Salemi Vera Maria Cury, Alexandre Leonardo Machado, Mady Charles. Miocardiopatia não compactada: uma visão atual. Arq. Bras. Cardiol. [Internet]. 2011.

5. Maron BJ, Towbin JA, Thiene G, et. al.. Contemporary definitions and classification of the cardiomyopathies. Clinical Cardiology. 2006;113(14):1807-16.

6. Oechslin E, Jenni R. Isolated left ventricular no-compaction: increasing recognition of this distinct, yet unclassified cardiomyopathy. Eur J Echocardiogr. 2002;3(4):250-1.

7. Junior, H P. Ferreira, M C F. Noncompaction of ventricular myocardium: case report with autopsy. J Bras Patol Med Lab. v.45 n.5. p.401-405.2009.

8. Jenni R, Oechslin E. et. al.. Echocardiographic and pathoanatomical characteristics of isolated left ventricular non-compaction: a step towards classification as a distinct cardiomyopathy. Heart. $2001 ; 86(6)$ :66671.

9. Avezum et. al.. Cardiovascular disease in South of America: current status and oportunities for prevention. Heart, v.95,n.18,p.1475-1482,2009.

10. Magalhães LP, Figueiredo MJO, Cintra FD, Saad EB, Kuniyoshi RR, Teixeira RA et. al.. II Diretrizes Brasileiras de Fibrilação Atrial. Arq. Bras. Cardiol. [Internet]. 2016.

1. Serviço de Cardiologia do HSI

2. Acadêmica de Medicina da Escola Bahiana de Medicina e Saúde Pública.

Endereço para correspondência:

albalima82011@hotmail.com 